\title{
Compositional Minimisation of Finite State Systems Using Interface Specifications ${ }^{1}$
}

\author{
Susanne Graf ${ }^{2}$, Bernhard Steffen ${ }^{3}$ and Gerald Lüttgen ${ }^{3}$ \\ ${ }^{2}$ VERIMAG, Monbonnot, France \\ ${ }^{3}$ Fakultät für Mathematik und Informatik, Universität Passau, Passau, Germany
}

Keywords: Bisimulation; Distributed system; Interface specification; Minimisation; State explosion problem

\begin{abstract}
We present a method for the compositional construction of the minimal transition system that represents the semantics of a given distributed system. Our aim is to control the state explosion caused by the interleavings of actions of communicating parallel components by reduction steps that exploit global communication constraints given in terms of interface specifications. The effect of the method, which is developed for bisimulation semantics here, depends on the structure of the distributed system under consideration, and the accuracy of the interface specifications. However, its correctness is independent of the correctness of the interface specifications provided by the program designer.
\end{abstract}

\section{Introduction}

Many tools for the automatic analysis or verification of finite state distributed systems are based on the construction of the global state graph of the system under consideration. Thus, they often fail because of the state explosion problem: the state space of a distributed system potentially increases exponentially in the number of its parallel components. To overcome this problem techniques have been developed in order to avoid the construction of the complete state graph. In this paper we present a method for the compositional minimisation of finite state

\footnotetext{
${ }^{1}$ A preliminary version appeared in Proceedings of $C A V^{\prime} 90$ - Second International Workshop on Computer-Aided Verification, Vol. 531 Lecture Notes in Computer Science, Springer, pp. 186-196, June 1990.

Correspondence and offprint requests to: Gerald Lüttgen, Fakultät für Mathematik und Informatik, Universität Passau, D-94030 Passau, Germany.
} 
distributed systems, which is practically motivated by the following observation: For the verification of a system it is usually sufficient to consider an abstraction of its global state graph, because numerous computations are irrelevant from the observer's point of view. Such abstractions often allow us to reduce the state graph drastically by collapsing semantically equivalent states to a single state without affecting the observable behaviour. For example, the so obtained minimisation of a complex communication protocol may be a simple buffer.

Let us refer to the size of the original state space of a system $S$ as its apparent complexity, and to the size of the minimised state space as its reduced complexity. The intention of our method is to avoid the apparent complexity by constructing the minimal system representation taking context information into account. Unfortunately, the straightforward idea to just successively combine and minimise the components of the system is not satisfactory, because such a "local" minimisation does not take context constraints into account and, therefore, may even lead to subsystems with a higher reduced complexity than the apparent complexity of the overall system. This is mainly due to the fact that parts need to be considered that can never be reached in the global context. Partial or loose specifications allow us to "cut off" these unreachable parts. We exploit this feature to take advantage of context information. Furthermore, we refer to the size of the maximal transition system that is encountered by our method as the algorithmic complexity.

Our method, called Reduction-Minimisation-Method (RMA-Method for short), is tailored for establishing $\mathrm{P} \models S p e c$, i.e. whether $\mathrm{P}$ satisfies the specification or property $\mathrm{Spec}$, when $\mathrm{P}$ is a system in standard concurrent form, i.e. $\mathrm{P}=$ $\left(p_{1}\left\|_{I_{1}} \ldots\right\|_{I_{n-1}} p_{n}\right)\langle L\rangle$, which is annotated by interface specifications, and $S p e c$ is consistent with the semantical equivalence under consideration, i.e. $P \models S p e c \Longleftrightarrow$ $Q=$ Spec if $P$ and $Q$ are semantically equivalent. To simplify the development of our theory, we assume that the processes $p_{i}$ are already given as transition systems and that $\|$ represents the parallel composition operator, $\langle L\rangle$ is a window or hiding operator that abstracts from the activities considered as internal by transforming them into the unobservable action $\tau$, and $I_{i}$ are interface specifications between $R_{i}=_{d f}\left(p_{1}\|\ldots\| p_{i}\right)$ and $Q_{i}=_{d f}\left(p_{i+1}\|\ldots\| p_{n}\right)$. Interface specifications are intended to describe supersets of the set of sequences that can be observed at the associated interfaces. We represent interface specifications as processes that have exclusively observable behaviour and cannot perform any internal step. A central result of the paper is that the branching structure of an interface specification is unimportant in our framework. Only its associated language has an impact. The point of our method is the successive construction of partially defined transition systems $P_{i}$, $1 \leq i \leq n$, with the following properties. First, $P_{i}$ is less specified than $R_{i}$, i.e. $P_{i}$ is smaller than $R_{i}$ with respect to the specification preorder $\leq$. This is the key for proving the correctness of the $\mathscr{R} \mathscr{M}$-Method, i.e. $\mathscr{P}_{n} \models S p e c$ implies $\mathrm{P}=S$ pec, if Spec is consistent with the kernel of $\leq$ (i.e. $\leq n \geq$ ). Second, $P_{n}$ is semantically equivalent to the full system $P$, whenever the interface specifications are correct. This guarantees the completeness of the $\mathscr{R} \mathscr{M}$-Method. Third, $P_{i}$ has the least number of states and transitions in its equivalence class.

In this paper, we are dealing with a refinement $\approx^{d}$ of observational equivalence [Mil89]. However, the method also adapts to other equivalences. Technically, we use a new operator, called reduction operator, in each step $P_{i}$ where $1 \leq i<n$. The purpose of this operator is to cut off all states and transitions of the "intermediate' transition systems that are not reachable according to the corresponding interface specification $I_{i}$. Although interface specifications, which should be pro- 
vided by the program designer, are in the focus of our approach, the correctness of the $\mathscr{R} \mathscr{M}$-Method does not depend on the correctness of these interface specifications. They are only used to "guide" the reduction. Thus, wrong interface specifications never lead to wrong results, i.e. if $\mathscr{P}_{n}=S p e c$ is valid, then P satisfies $S p e c$, too, provided that $S p e c$ is consistent with the kernel of $\leq$. Otherwise, if $\mathscr{P}_{n} \models S p e c$ is not provable, then $\mathrm{P}$ may satisfy Spec or not. Therefore, wrong interface specifications may only prevent a successful verification of a valid statement. However, the $\mathscr{R} \mathscr{M}$-Method is complete in the following sense: If all the considered interface specifications are correct and if Spec is a $\approx^{d}$-consistent property, then $\mathscr{P}_{n} \models S p e c \Longleftrightarrow \mathrm{P} \models$ Spec. It should be noted that the total definedness of $\mathscr{P}_{n}$ already implies the semantical equivalence of $\mathscr{P}_{n}$ and $\mathrm{P}$ and, therefore, the completeness for all $\approx^{d}$-consistent properties. This criterion is sufficient for most practical applications.

\subsection{Related Work}

Great efforts have already been made in order to avoid the construction of a complete state graph, and therefore to avoid the state explosion problem. Roughly, the proposed methods can be split into two categories, compositional verification and compositional minimisation. In the first methods, the global system need not be considered at all, whereas in the second ones, a minimal semantically equivalent representation of the global system is constructed.

A pure approach to compositional verification has been proposed by Winskel in [Win90], where rules are given to decompose assertions of the form $P \models \Phi$ depending on the syntax of the program $\mathrm{P}$ and the formula $\Phi$. Unfortunately, the decomposition rules for processes involving the parallel operator are very restricted. In order to deal with the problems that arise from parallel compositions, Pnueli [Pnu90] proposed a "conditional" inference system where assertions of the form $\phi \mathrm{P} \psi$ can be derived, meaning that the program $\mathrm{P}$ satisfies the property $\psi$ if its environment satisfies $\phi$. This inference system has been used by Shurek and Grumberg in [ShG90], where a semi-automatic modular verification method is presented which, like ours, is based on "guesses" for context specifications. However, in contrast to our method, it requires a separate proof of the correctness of these guesses. Josko [Jos87] presented a method, where the assumptions on the environment are expressed by formulas, which must be proved in a separate step. However, this algorithm is exponential in the size of the assumptions. Other methods try to avoid the state explosion problem using preorders [GoW91, Pe193, Va193] where unnecessary interleavings of actions are eliminated during the construction. In [LSW94] a constraint-oriented state-based proof methodology is presented which exploits compositionality and abstraction for the reduction of the (possibly infinite) verification problem under consideration. There, Modal Transition Systems are used for fine-granular, loose state-based specifications of constraints.

Halbwachs et al. [BFH90] proposed a method of the second category. It constructs directly a minimal transition system with respect to bisimulation by successive refinement of a single state. In this method, symbolic computation is used in order to keep the expressions small. Clarke et al. [CLM89] exploit the knowledge about the alphabet of interest in order to abstract and minimise the system's components. By using $\langle L\rangle$ operators together with an elementary rule for distributing them over the parallel operator (see Proposi- 
tion 2.5) our method covers this approach. Larsen and Thomsen [LaT88], and Walker [Wal88] use partial specifications in order to take context constraints into account. Our method is an elaboration of theirs. It uses a more appropriate preorder and defines a concrete strategy for (semi-)automatic proofs where the required user support is kept to a minimum. Also Vaandrager [Vaa90] observes that in most situations partial information about the traces of processes is sufficient to prove that part of a specification is redundant and can be omitted.

All mentioned techniques can be accompanied by abstraction which may dramatically reduce the complexity [CGL92, DGG93, LGS95].

\section{General Notions}

Our framework is based on processes (systems) as labelled transition systems extended by an undefinedness predicate on states. Processes can be structured by means of parallel composition and hiding, thus allowing a hierarchical treatment.

A labelled transition system is a quadruple $(S, \mathscr{A} \cup\{\tau\}, \longrightarrow, \uparrow)$ where $S$ is a finite set of processes or states, $\mathscr{A}$ is a finite alphabet of observable actions, and $\tau$ represents an internal or unobservable action not in $\mathscr{A}$, the relation $\longrightarrow \subseteq S \times(\mathscr{A} \cup\{\tau\}) \times S$ is a transition relation, and $\uparrow \subseteq S \times 2^{(\mathscr{A} \cup\{\tau\})}$ is a predicate expressing guarded undefinedness.

Typically, $S$ is a set of program states, and the relationship $(p, a, q) \in \longrightarrow$, or $p \stackrel{a}{\longrightarrow} q$ for short, indicates that $p$ can evolve to $q$ under the observation of $a$. We write $p \stackrel{a}{\rightarrow}$ if $\exists q . p \stackrel{a}{\rightarrow} q$. Finally, $p \uparrow a$ expresses that an $a$-transition would allow $p$ to enter an undefined state. We say that $p$ is a-undefined in this case. Processes are rooted extended transition systems, i.e. they consist of an extended transition system and a designated start state.

Definition 2.1. Let $T=(S, \mathscr{A} \cup\{\tau\}, \longrightarrow, \uparrow)$ be an extended transition system. A process is a tuple $\left(S_{p}, \mathscr{A}_{p} \cup\{\tau\}, \longrightarrow_{p}, \uparrow_{p}, p\right)$ for a state $p \in S$ where $S_{p}$ is the set of states that are reachable from $p$ in $T, \mathscr{A}_{p}={ }_{d f} \mathscr{A}$, and $\longrightarrow_{p}$ and $\uparrow_{p}$ are $\longrightarrow$ and $\uparrow$ restricted to $S_{p}$, respectively. $p$ is called start state of the process. The set of all processes is denoted by $\mathscr{P}$.

We write $p$ for $\left(S_{p}, \mathscr{A}_{p} \cup\{\tau\}, \longrightarrow_{p}, \uparrow_{p}, p\right)$ and say that a process is totally defined if its undefinedness predicate $\uparrow_{p}$ is empty. Moreover, if $p, q \in \mathscr{P}$ are identical up to renamings of states, we call $p$ and $q$ isomorphic, in signs $p \cong q$. In the remainder, we also need weak versions of transition relation and undefinedness predicate which abstract from the invisible action $\tau$.

Definition 2.2. Let $(S, \mathscr{A} \cup\{\tau\}, \longrightarrow, \uparrow)$ be an extended transition system. The weak transition relation $\Longrightarrow \subseteq S \times(\mathscr{A} \cup\{\epsilon\}) \times S$ and the weak undefinedness predicate $\Uparrow \subseteq S \times 2^{(\mathscr{A} \cup\{\epsilon\})}$ are defined as the least relations satisfying for all $p, q \in S$ and all $a \in \mathscr{A}:$

1. $p \stackrel{\tau}{\longrightarrow} \stackrel{a}{\longrightarrow} \stackrel{\tau}{\longrightarrow} *$ implies $p \stackrel{a}{\longrightarrow} q$

3. $q \uparrow a$ and $p \stackrel{\epsilon}{\Longrightarrow} q$ implies $p \Uparrow a$

2. $p \stackrel{\tau}{\longrightarrow}$ * $q$ implies $p \stackrel{\epsilon}{\longrightarrow} q$

5. $q \Uparrow \epsilon$ and $p \stackrel{a}{\Longrightarrow} q$ implies $p \Uparrow a$

4. $q \uparrow \tau$ and $p \stackrel{\epsilon}{\Longrightarrow} q$ implies $p \Uparrow \epsilon$

6. $p \Uparrow \epsilon$ implies $p \Uparrow a$

Now, we are able to define the language of processes. 
Definition 2.3. The language, $\mathscr{L}(p)$, of a partially defined process $p$ is defined as the least fixed point of the following equation system:

$$
\begin{aligned}
& \mathscr{L}(p)= \begin{cases}\mathscr{A}_{p}^{*} & \text { if } p \Uparrow \varepsilon \\
\bigcup^{\{}\left\{a \cdot \mathscr{L}_{a}(p) \mid \mathscr{L}_{a}(p) \neq \emptyset\right\} \cup\{\varepsilon\} & \text { otherwise }\end{cases} \\
& \mathscr{L}_{a}(p)= \begin{cases}\mathscr{A}_{p}^{*} & \text { if } p \Uparrow a \\
\bigcup\left\{\mathscr{L}\left(p^{\prime}\right) \mid p \stackrel{a}{\Longrightarrow} p^{\prime}\right\} & \text { otherwise }\end{cases}
\end{aligned}
$$

Note that this definition is standard for totally defined processes. For undefined processes we assume the "worst case" that the language contains all possible sequences of actions.

\subsection{Parallel Composition and Hiding}

We now introduce a binary parallel operator $\|$ and unary hiding or window operators $\langle L\rangle$ on processes, where $L$ is the set of actions remaining visible. Intuitively, $p \| q$ is the parallel composition of the processes $p$ and $q$ with synchronisation of the actions common to both of their alphabets and interleaving of the others, and $p\langle L\rangle$ is the process in which only the actions in $L$ are observable.

Definition 2.4. Let $p=\left(S_{p}, \mathscr{A}_{p} \cup\{\tau\}, \longrightarrow_{p}, \uparrow_{p}, p\right), q=\left(S_{q}, \mathscr{A}_{q} \cup\{\tau\}, \longrightarrow_{q}, \uparrow_{q}, q\right) \in \mathscr{P}$, let $p^{\prime}, p^{\prime \prime} \in S_{p}, q^{\prime}, q^{\prime \prime} \in S_{q}$, and let $L$ be a set of visible actions. We define the alphabets of the processes $p\langle L\rangle$ and $p \| q$ by $\mathscr{A}_{p\langle L\rangle}={ }_{d f} \mathscr{A}_{p} \cap L$ and $\mathscr{A}_{p \| q}={ }_{d f}$ $\mathscr{A}_{p} \cup \mathscr{A}_{q}$, respectively. Their state sets are defined as the subsets of states of $\left\{p^{\prime}\langle L\rangle \mid p^{\prime} \in S_{p}\right\}$ and $\left\{p^{\prime} \| q^{\prime} \mid p^{\prime} \in S_{p}, q^{\prime} \in S_{q}\right\}$ which are reachable from the initial states $p\langle L\rangle$ and $p \| q$, respectively, according to the transition relations defined in Plotkin style notation below. Moreover, we define rules for the undefinedness predicates of $p\langle L\rangle$ and $p \| q$.
1. $\frac{p^{\prime} \stackrel{a}{\longrightarrow} p p^{\prime \prime}}{p^{\prime}\langle L\rangle \stackrel{a}{\longrightarrow} p\langle L\rangle p^{\prime \prime}\langle L\rangle} \quad a \in L$
6. $\frac{p^{\prime} \uparrow_{p} a}{p^{\prime}\langle L\rangle \uparrow_{p\langle L\rangle} a} \quad a \in L$
2. $\frac{p^{\prime} \stackrel{a}{\longrightarrow} p p^{\prime \prime}}{p^{\prime}\langle L\rangle \stackrel{\tau}{\longrightarrow} p\langle L\rangle p^{\prime \prime}\langle L\rangle} \quad a \notin L$
7. $\frac{p^{\prime} \uparrow_{p} a}{p^{\prime}\langle L\rangle \uparrow_{p\langle L\rangle} \tau} \quad a \notin L$
3. $\frac{p^{\prime} \stackrel{a}{\rightarrow} p p^{\prime \prime}}{p^{\prime}\left\|q^{\prime} \stackrel{a}{\rightarrow} p\right\| q p^{\prime \prime} \| q^{\prime}} \quad a \notin \mathscr{A}_{q}$
8. $\quad \frac{p^{\prime} \uparrow_{p} a}{\left(p^{\prime} \| q^{\prime}\right) \uparrow_{p \|_{q}} a} \quad a \notin \mathscr{A}_{q}$ or $q^{\prime} \stackrel{a}{\longrightarrow} q$
4. $\frac{q^{\prime} \stackrel{a}{\longrightarrow} q q^{\prime \prime}}{p^{\prime}\left\|q^{\prime} \stackrel{a}{\rightarrow} p\right\| q p^{\prime} \| q^{\prime \prime}} \quad a \notin \mathscr{A}_{p}$
9. $\quad \frac{q^{\prime} \uparrow_{q} a}{\left(p^{\prime} \| q^{\prime}\right) \uparrow_{p \| q} a} \quad a \notin \mathscr{A}_{p}$ or $p^{\prime} \stackrel{a}{\longrightarrow} p$
5. $\frac{p^{\prime} \stackrel{a}{\longrightarrow} p p^{\prime \prime} \quad q^{\prime} \stackrel{a}{\longrightarrow} q q^{\prime \prime}}{p^{\prime}\left\|q^{\prime} \stackrel{a}{\longrightarrow} p\right\| q p^{\prime \prime} \| q^{\prime \prime}} \quad a \neq \tau$
10. $\frac{p^{\prime} \uparrow_{p} a q^{\prime} \uparrow_{q} a}{\left(p^{\prime} \| q^{\prime}\right) \uparrow_{p \| q} a}$

Thus, $p^{\prime} \uparrow_{p} a\left(q^{\prime} \uparrow_{q} a\right)$ implies $\left(p^{\prime} \| q^{\prime}\right) \uparrow_{p \|_{q}} a$, whenever $q^{\prime}\left(p^{\prime}\right)$ does not preempt the execution of $a$, i.e. whenever $a \notin \mathscr{A}_{q}$ or $q^{\prime} \stackrel{a}{\longrightarrow} q\left(a \notin \mathscr{A}_{p}\right.$ or $\left.p^{\prime} \stackrel{a}{\longrightarrow} p\right)$. Since the parallel operator is associative and commutative, processes in standard concurrent form $\left(p_{1}\|\ldots\| p_{n}\right)\langle L\rangle$ are well-defined. Usually, compositional minimisation techniques localise global hiding information based on the following observation.

Proposition 2.5. Let $p, q \in \mathscr{P}$ and $L, L^{\prime}$ be sets of visible actions satisfying $L^{\prime} \supseteq$ $L \cup\left(\mathscr{A}_{p} \cap \mathscr{A}_{q}\right)$. Then $(p \| q)\langle L\rangle \cong\left(p\left\langle L^{\prime}\right\rangle \| q\right)\langle L\rangle$. 


\subsection{Semantical Equivalence and Preorder}

We define a semantical equivalence on extended labelled transition systems in terms of observational equivalence [Mil89] and establish a specification-implementation relation in terms of a preorder, which is compatible with this semantics.

Definition 2.6. Let $(S, \mathscr{A} \cup\{\tau\}, \longrightarrow, \uparrow)$ be an extended transition system. Then $\approx^{d}$ is the union of all relations $R \subseteq S \times S$ satisfying that $(p, q) \in R$ implies for all $a \in \mathscr{A} \cup\{\epsilon\}:$

1. $p \Uparrow a$ if and only if $q \Uparrow a$,

2. $p \stackrel{a}{\Longrightarrow} p^{\prime}$ implies $\exists q^{\prime} \cdot q \stackrel{a}{\Longrightarrow} q^{\prime} \wedge\left(p^{\prime}, q^{\prime}\right) \in R$, and

3. $q \stackrel{a}{\Longrightarrow} q^{\prime}$ implies $\exists p^{\prime} . p \stackrel{a}{\Longrightarrow} p^{\prime} \wedge\left(p^{\prime}, q^{\prime}\right) \in R$.

Two processes $p, q \in \mathscr{P}$ with the same alphabet are equivalent if and only if their extended transition systems can be combined into one extended transition system and the states $p$ and $q$ are equivalent according to the above definition. The following preorder, which intuitively defines a "less defined than" relation between processes, is the basis of the framework in which we establish the correctness of our $\mathscr{R} \mathscr{U}$-Method (cf. [C1S90]).

Definition 2.7. Let $(S, \mathscr{A} \cup\{\tau\}, \longrightarrow, \uparrow)$ be an extended transition system. The specification preorder $\leq$ is the union of all relations $R \subseteq S \times S$ satisfying $(p, q) \in R$ implies for all $a \in \mathscr{A} \cup\{\epsilon\}$ with $\neg(p \Uparrow a)$ :

1. $\neg(q \Uparrow a)$,

2. $p \stackrel{a}{\Longrightarrow} p^{\prime}$ implies $\exists q^{\prime} \cdot q \stackrel{a}{\Longrightarrow} q^{\prime} \wedge\left(p^{\prime}, q^{\prime}\right) \in R$, and

3. $q \stackrel{a}{\Longrightarrow} q^{\prime}$ implies $\exists p^{\prime} . p \stackrel{a}{\Longrightarrow} p^{\prime} \wedge\left(p^{\prime}, q^{\prime}\right) \in R$.

In our context, this preorder is used as a specification-implementation relation: a partial specification $p$ is met by an implementation $q$ if and only if $p \leq q$. Observational equivalence $\approx$ and our specification-preorder $\preceq$ induce slightly different semantics on processes. However, $\approx^{d}$ is a refinement of both, and all relations coincide for totally defined processes. Of special importance for our compositional minimisation method is the preservation of $\preceq$ and $\approx^{d}$ by the operators $\|$ and $\langle L\rangle$.

\subsection{Interface Specifications}

We introduce the notion of interface specification together with a notion of correctness, which guarantees the success of the $\mathscr{R} \mathscr{M}$-Method. These notions concentrate on the set of observable sequences that may pass the interface. Thus, the exact specification of the interface between processes $p$ and $q$ is the language of $(p \| q)\left\langle\mathscr{A}_{p} \cap \mathscr{A}_{q}\right\rangle$, i.e. its set of observable sequences.

We are going to use interface specifications in order to express context constraints. Therefore, interface specifications are correct or safe if the corresponding exact interface specification is more constraint. This motivates the following definition.

Definition 2.8. Let $p, q \in \mathscr{P}$. A totally defined process $I \in \mathscr{P}$ without $\tau$-transitions is an interface specification for $p$ iff $\mathscr{A}_{I} \subseteq \mathscr{A}_{p}$. It is an interface specification for $p$ 
and $q$ iff $\mathscr{A}_{I}=\mathscr{A}_{p} \cap \mathscr{A}_{q}$. An interface specification $I$ for $p$ and $q$ is called correct for $p$ and $q$ iff $\mathscr{L}\left((p \| q)\left\langle\mathscr{A}_{p} \cap \mathscr{A}_{q}\right\rangle\right) \subseteq \mathscr{L}(I)$. The set of all interface specifications for $p$ is denoted by $\mathscr{I}(p)$, and the set of all correct interface specifications for $p$ and $q$ by $\mathscr{I}(p, q)$. Finally, we write $\mathscr{I}$ for $\bigcup\{\mathscr{I}(p) \mid p \in \mathscr{P}\}$, i.e. the set of all totally defined processes without $\tau$-transitions.

Theorem 3.3 shows that these language-based definitions are adequate for our purposes.

\section{Reduction Operators}

In this section we propose a general notion of reduction operators and a special instance of it, which is suitable for our purposes.

Definition 3.1. A partial mapping $\Pi: \mathscr{I} \times \mathscr{P} \longrightarrow \mathscr{P}$ is called reduction operator if

(i) $\forall p \in \mathscr{P}, I \in \mathscr{I}(p)$. $\Pi(I, p) \leq p$ (Correctness for arbitrary interfaces)

(ii) $\forall p, q \in \mathscr{P}, I \in \mathscr{I}(p, q)$. $\Pi(I, p)\left\|q \approx^{d} p\right\| q$ (Context preservation for correct interfaces)

(iii) $\forall p \in \mathscr{P}, I \in \mathscr{I}(p) .\left|S_{\Pi(I, p)}\right| \leq\left|S_{p}\right|$ and $\left|\longrightarrow_{\Pi(I, p)}\right| \leq\left|\longrightarrow_{p}\right|$ (Reduction)

In the following we often write $\Pi_{I}(p)$ instead of $\Pi(I, p)$.

Intuitively, a reduction operator $\Pi$ should eliminate those states and transitions of a process $p \in \mathscr{P}$ which are not reachable in each global context satisfying the interface specification $I \in \mathscr{F}(p)$. This 'algorithmic' intuition guarantees the first two conditions, which are essential for a sensible notion of reduction operator: the first condition is a correctness requirement. The reduction always yields a process which behaves on its defined part as $p$. The second condition guarantees that the reduction does not affect the behaviour of $p$ in a context satisfying the (correct) interface specification. Finally, the third condition reflects the primary intuition of reduction: the number of states and transitions should be reduced. This is by no means guaranteed by a decrease in the preorder! The following operator $\Pi$ satisfies the conditions of Definition 3.1.

Definition 3.2. The reduction operator $\bar{\Pi}$ is defined by the mapping $(I, p) \longmapsto$ $\bar{\Pi}(I, p)={ }_{d f}(S, \mathscr{A} \cup\{\tau\}, \longrightarrow, \uparrow, p)$ for $I=\left(S_{I}, \mathscr{A}_{I}, \longrightarrow{ }_{I}, \emptyset, I\right) \in \mathscr{I}(p)$ and $p=$ $\left(S_{p}, \mathscr{A}_{p} \cup\{\tau\}, \longrightarrow_{p}, \uparrow_{p}, p\right) \in \mathscr{P}$ where

1. $S=\left\{q \in S_{p} \mid \exists i \in S_{I} . q \| i \in S_{p \| I}\right\}$,

2. $\mathscr{A}=\mathscr{A}_{p}$,

3. $\forall q, q^{\prime} \in S, a \in \mathscr{A} \cup\{\tau\} . q \stackrel{a}{\longrightarrow} q^{\prime}$ iff $\exists i, i^{\prime} \in S_{I} . q\|i \stackrel{a}{\longrightarrow} p\| I q^{\prime} \| i^{\prime},{ }^{\prime}$

4. $\forall q \in S . q \uparrow \tau$ iff $q \uparrow_{p} \tau$, and

5. $\forall q \in S, a \in \mathscr{A} \cdot q \uparrow a$ iff $q \uparrow_{p} a$ or $\left(\exists q^{\prime} \in S_{p} . q \stackrel{a}{\longrightarrow} p q^{\prime}\right.$ and $\left.\exists q^{\prime} \in S . q \stackrel{a}{\longrightarrow} q^{\prime}\right)$.

In conformance with Definition 3.1 we also write $\bar{\Pi}_{I}(p)$ for $\bar{\Pi}(I, p)$.

\footnotetext{
$\overline{1 \text { This implies by the definitions of } S_{p \| I}}$ and $\longrightarrow p \mid I$ according to Definition 2.1 that $q \| i$ is reachable in $p \| I$.
} 
The only difference between $\bar{\Pi}(I, p)$ and the projection of $p \| I$ onto $p$ concerns the undefinedness predicate: $\Pi(I, p)$ inherits all undefinednesses from $p$, and new ones are introduced where transitions of $p$ have been cut off by $I$. The point of the reduction operator is that for correct interface specifications this second kind of undefinedness disappears again in the full context $\bar{\Pi}(I, p) \| q$. This holds, because if an $a$-transition of $p$ has been replaced by $\uparrow a$, this predicate disappears again in $\bar{\Pi}(I, p) \| q$ exactly if $q$, in its corresponding state, preempts the execution of an $a$-transition. Thus, the presence of an $\uparrow a$ in $\bar{\Pi}(I, p) \| q$ indicates a fault in the interface specification, whenever $p$ and $q$ are totally defined processes. Note that it is possible that $\bar{\Pi}(I, p) \| q$ is totally defined, although $I$ is not correct for $p$ and $q$. This is the case if the incorrect parts of $I$ need not be considered for the reduction. Our reduction operator satisfies representation independence which states that not the branching structure of an interface specification but only its language is important.

Theorem 3.3. (Representation Independence) For all $p \in \mathscr{P}$ and for all $I, I^{\prime} \in \mathscr{I}(p)$ we have: $\mathscr{L}(I)=\mathscr{L}\left(I^{\prime}\right)$ implies $\bar{\Pi}_{I}(p) \cong \bar{\Pi}_{I^{\prime}}(p)$.

\section{Minimisation Method}

Now, we develop the $\mathscr{R} \mathscr{M}$-Method which compositionally minimises finite state distributed systems, on top of a reduction operator $\Pi$. It is tailored to deal with systems of the form $\mathrm{P}=\left(p_{1}\|\ldots\| p_{n}\right)\langle L\rangle$. The $\mathscr{R} \mathscr{M}$-Method expects the system $\mathrm{P}$ to be annotated with interface specifications for the right hand process and the left hand process of the parallel operator they are attached to: $\left(p_{1}\left\|_{I_{1}} p_{2}\right\|_{I_{2}} \ldots \|_{I_{n-1}} p_{n}\right)\langle L\rangle$. It proceeds by successively constructing transition systems $\mathscr{P}_{i}$ as follows:

- $\mathscr{P}_{1}={ }_{d f} \mathscr{M}\left(\Pi_{I_{1}}\left(\mathscr{M}\left(p_{1}\left\langle\mathscr{A}_{I_{1}} \cup L\right\rangle\right)\right)\right)$,

- $\mathscr{P}_{i}={ }_{d f} \mathscr{M}\left(\Pi_{I_{i}}\left(\mathscr{M}\left(\left(\mathscr{P}_{i-1} \| p_{i}\right)\left\langle\mathscr{A}_{I_{i}} \cup L\right\rangle\right)\right)\right)$ for $2 \leq i \leq n-1$, and

- $\mathscr{P}_{n}=d f \mathscr{M}\left(\left(\mathscr{P}_{n-1} \| p_{n}\right)\langle L\rangle\right)$.

In order to avoid unnecessarily large intermediate transition systems, it is important to minimise all the intermediate constructions as it is done above. Note that this method covers the naive method (only using $\mathscr{M}$ ) and methods which only consider the correspondence of the parallel and the window operator (see Proposition 2.5). The new additional power of the $\mathscr{R} \mathscr{M}$-Method is due to the reduction operator $\mathrm{II}$ which minimises all intermediate transition systems $\mathscr{P}_{i}$ according to global constraints specified in terms of the interface specifications $I_{i}$ (for $1 \leq i \leq n-1$ ). We obtain the following correctness result, which is independent of the correctness of the interface specifications.

Theorem 4.1. (Correctness) $\mathscr{P}_{n} \leq \mathrm{P}$ holds.

This is already enough to guarantee the correctness of the $\mathscr{R} \mathscr{M}$-Method, i.e. any property consistent with the kernel of $\leq$ that holds on $\mathscr{P}_{n}$ is also valid on P. Thus, faulty interface specifications never allow us to establish wrong properties. They may only prevent their satisfaction on the reduced system. Also, the correctness of the interface specifications is already sufficient to guarantee the success of the method, i.e. the reduction is consistent with the semantical equivalence.

Theorem 4.2. (Completeness) $\left(\forall j \leq n . I_{j} \in \mathscr{I}\left(p_{1}\|\ldots\| p_{j}, Q_{j}\right)\right)$ implies $\mathscr{P}_{n} \approx^{d} \mathrm{P}$. 
In practice, $\mathrm{P}$ is usually totally defined. Then the proof of $\mathscr{P}_{n} \approx d$ P reduces to the verification of the total definedness of $\mathscr{P}_{n}$.

Corollary 4.3. Whenever $\mathrm{P}$ is totally defined, we have: $\mathscr{P}_{n} \approx^{d} \mathrm{P}$ iff $\mathscr{P}_{n}$ is totally defined.

\section{Conclusions}

We have presented a method for the compositional minimisation of finite state distributed systems which is intended to avoid the state explosion problem. This method can be used to support the verification of any property that is consistent with $\approx^{d}$ and is implemented as part of the METAFrame environment [SMC96] and the Aldebaran verification tool [Fer88]. The effect of this method depends on interface specifications, which we assume to be given by the program designer. However, the correctness of our method does not depend on the correctness of these interface specifications. This is very important, because it allows the designer to simply "guess" interface specifications, while maintaining the reliability of a successful verification.

A full version of this paper is available electronically [GSL96* ]. It contains an efficient algorithm for $\bar{\Pi}$ and illustrating examples which show the power of our method by minimising a system whose apparent complexity is exponential, but its algorithmic and reduced complexities are linear. Finally, the electronic version includes all challenging proofs and a more detailed discussion of related work.

\section{Acknowledgements}

We want to thank Rance Cleaveland, Ernst-Rüdiger Olderog, Dirk Koschützki, and the anonymous referees for their valuable comments and suggestions.

\section{References}

[BFH90] Bouajjani, A., Fernandez, J.-C. and Halbwachs, N.: Minimal model generation. In Workshop on Automatic Verification '90, volume 531 of LNCS, 1990.

[CGL92] Clarke, E., Grumberg, O. and Long, D.: Model checking and abstraction. In Symp. Principles of Programming Languages '92, 1992.

[CLM89] Clarke, E., Long, D. and McMillan, K.: Compositional model checking. Proc. IEEE Symp. Logic in Computer Science, pages 353-362, 1989.

[ClS90] Cleaveland, R. and Steffen, B.: A preorder for partial process specifications. In Proceedings of CONCUR'90, Amsterdam (Netherlands), volume 458 of LNCS, 1990.

[DGG93] Dams, D., Grumberg, O. and Gerth, R.: Generation of reduced models for checking fragments of CTL. In Proceedings of the International Conference on Computer-Aided Verification (CAV'93), volume 697 of LNCS, pages 479-490, 1993.

[Fer88] Fernandez, J.-C.: Aldébaran: Un Système de Vérification par Réduction de Processus Communicants. PhD thesis, Université de Grenoble, 1988.

[GoW91] Godefroid, P. and Wolper, P.: Using partial orders for the efficient verification of deadlock freedom and safety properties. In Proceedings of the International Workshop on Computer-Aided Verification ( $C A V^{\prime} 91$ ), volume 575 of $L N C S$, pages $332-342,1991$.

[GSL96*] Graf, S., Steffen, B. and Lüttgen, G.: Compositional Minimisation of Finite State Systems Using Interface Specifications, Formal Aspects of Computing 8(E):xxx-xxx.

\footnotetext{
* This paper can be retrieved by downloading the (compressed PostScript) file: FACj-8E-pp xxx-Xxx ps. $Z$ which can be found in the directory pub/fac on the ftp.cs.man.ac.uk
} 
[Jos87] Josko, B.: MCTL - an extension of CTL for modular verification of concurrent systems. In Workshop on Temporal Logic in Specification, volume 398 of LNCS, 1987.

[LGS95] Loiseaux, C., Graf, S., Sifakis, J., Bouajjani, A. and Bensalem, S.: Property preserving abstractions for the verification of concurrent systems. Formal Methods in System Design, Vol 6, Iss 1, 1995.

[LSW94] Larsen, K. G., Steffen, B. and Weise, C.: A constraint oriented proof methodology based on modal transition systems. In BRICS Notes 94-6, December 1994.

[LaT88] Larsen, K. G. and Thomsen, B.: Compositional proofs by partial specification of processes. Proc. IEEE Symp. Logic in Computer Science, 1988.

[Mil89] Milner, R.: Communication and Concurrency. Prentice Hall, 1989.

[Pel93] Peled, D.: All from one, one for all: on model checking using representatives. In Proceedings of the International Conference on Computer Aided Verification (CAV'93), volume 697 of $L N C S, 1993$.

[Pnu90] Pnueli, A.: In transition from global to modular temporal reasoning about programs. In Logics and Models for Concurrent Systems, volume 13 of NATO ASI Series F. Springer Verlag, 1990.

[ShG90] Shurek, G. and Grumberg, O.: The modular framework of computer-aided verification. In Workshop on Automatic Verification '90, volume 531 of LNCS, pages 214-223, 1990.

[SMC96] Steffen, B., Margaria, T. and Claßen, A.: Heterogeneous analysis and verification for distributed systems. Software-Concepts and Tools, 17:13-25, 1996.

[Vaa90] Vaandrager, F. W.: Some observations on redundancy in a context. In J.C.M. Baeten, editor, Applications of Process Algebra, volume 17 of Cambridge Tracts in Theoretical Computer Science, pages 237-260. Cambridge University Press, 1990.

[Va193] Valmari, A.: On-the-fly verification with stubborn sets. In Proceedings of the International Conference on Computer Aided Verification (CAV'93), volume 697 of LNCS, pages 397408, 1993.

[Wa188] Walker, D. J.: Bisimulation and divergence in CCS. Proc. IEEE Symp. Logic in Computer Science, 1988.

[Win90] Winskel, G.: Compositional checking of validity on finite state processes. In Workshop on Theories of Communication, CONCUR, volume 458 of LNCS, 1990.

Received February 1995

Accepted in revised form May 1996 by A. J. R. G. Milner 